\title{
Dysmotility Symptoms and Weight Change: A Psychiatric Perspective
}

TO THE EDITOR: In a recent issue of the Journal of Neurogastroenterology and Motility, we were very interested in the article by Eslick et al ${ }^{1}$ entitled "Dysmotility symptoms are independently associated with weight change: a population-based study of Australian adults," in which the investigators assessed the associations between gastrointestinal (GI) symptoms and weight change (loss or gain). They reported that specific dysmotility symptoms are independently predictive of both weight loss and weight gain, and that different pathogenic mechanisms may be involved. This study is well designed, but we would like to comment on several of the factors that can affect both GI symptoms and weight change, as these may shed new light on the author's interpretations.

The authors state that $54 \%$ of participants were women, and mean age was not given in the study. The average menopausal period begins at 52 years. However, premature menopause may occur earlier. Prior studies have shown that body weight changes during the menopause. ${ }^{2}$ Furthermore, GI symptoms vary according to the phase of the menstrual cycle. ${ }^{3}$ For all these reasons, the menstrual status (cycle) and reproductive functions of participants should be considered in their study.

Obesity and body weight changes are frequently associated a number of psychiatric disorders, especially mood disorders, anorexia nervosa, and bulimia nervosa. Individuals with major depressive disorder have an approximately 50\% higher risk of developing obesity, when compared to the general population. ${ }^{4}$ Conversely, overweight and obesity have been reported to increase the risk of onset of significant depressive and anxiety symptoms. ${ }^{5,6}$

In addition to the above diseases, physical exercise, stress exposure, and lifestyle-related health behaviors are also important factors that could alter body weight and GI symptoms. ${ }^{4-7}$ The authors did not mention any of the above as potential contributing factors.

Lastly, several medications such as especially antidepressants and antipsychotics have been shown to affect body weight. Besides alcohol and smoking, substance abuse also affects body weight. ${ }^{8}$

In conclusion, elaboration about the menopause situation, menstrual cycle, depression and anxiety levels, psychiatric disorders, physical exercise levels, lifestyle-related health behaviors, medication use, and substance abuse of the study participants will certainly provide clearer information for readers.

\section{Cemil Celik, Barbaros Ozdemir, and Taner Oznur Department of Psychiatry, Gulhane Military Medical Faculty, Ankara, Turkey}

1. Eslick GD, Howell SC, Talley NJ. Dysmotility symptoms are independently associated with weight change: a population-based study of Australian Adults. J Neurogastroenterol Motil 2015;21:603-611.

2. Matthews KA, Abrams B, Crawford S, et al. Body mass index in mid-life women: relative influence of menopause, hormone use, and ethnicity. Int J Obes Relat Metab Disord 2001;25:863-873.

3. Bharadwaj S, Barber MD, Graff LA, Shen B. Symptomatology of irritable bowel syndrome and inflammatory bowel disease during the menstrual cycle. Gastroenterol Rep (Oxf) 2015;3:185-193.

4. Mansur RB, Brietzke E, McIntyre RS. Is there a "metabolic-mood syndrome"? A review of the relationship between obesity and mood disorders. Neurosci Biobehav Rev 2015;52:89-104.

5. Luppino FS, de Wit LM, Bouvy PF, et al. Overweight, obesity, and depression: a systematic review and meta-analysis of longitudinal studies. Arch Gen Psychiatry 2010;67:220-229.

6. Kubzansky LD, Gilthorpe MS, Goodman E. A prospective study of psychological distress and weight status in adolescents/young adults. Ann Behav Med 2012;43:219-228

7. Richardson T, Elliott P, Roberts R.The relationship between personal 
unsecured debt and mental and physical health: a systematic review and meta-analysis. Clin Psychol Rev 2013;33:1148-1162.

8. Dent R, Blackmore A, Peterson J, et al. Changes in body weight and psychotropic drugs: a systematic synthesis of the literature. PLoS One 2012; $7: e 36889$

\section{Conflicts of interest: None.}

